\title{
Mechanical Properties and Failure Mechanism of Sandstone with Mudstone Interlayer
}

\author{
Chengxiang Wang ${ }^{1}$, Huailiang Kou${ }^{1}$, Wei Han ${ }^{2 *}$ \\ ${ }^{1}$ School of Civil Engineering and Architecture, Shandong University of Science and Technology, Qingdao, Shandong, 266590, \\ China \\ ${ }^{2}$ Graduate School of Engineering, Nagasaki University, Nagasaki 852-8521, Japan.
}

\begin{abstract}
In order to study the strength and failure characteristics of sandstone with mudstone interlayer. According to mudstone interlayer dip and distribution form, it can be divided into three kinds of sandstone: sandstone with horizontal mudstone interlayer is Class I; sandstone with inclined mudstone interlayer is Class II; sandstone with irregular distribution mudstone is Class III. The three kinds of sandstone uniaxial test were carried out and combined with the theory of layered rock mass failure, it revealed mechanical properties and failure mechanism of sandstone with mudstone interlayer. The results show that: crack generated from interface in Class I and II and crack generated from irregular distribution mudstone interlayer in Class III; The sandstone with higher strength is destroyed before the mudstone interlayer; sandstone at unbonded interface is failure before sandstone at bonded interface; Three kinds of sandstone vary in compressive strength, Class II is greater than Class III while Class III is greater than Class I.
\end{abstract}

\section{Introduction}

Layered sandstone exists widely in nature. Sandstone often contains mudstone interlayer and it has a great impact on rock strength and failure mode. There are direct or indirect relations between the stability of surrounding rock and the dynamic phenomena in mining engineering, such as rock burst and rock fragmentation. Therefore, the study of rocks with weak interbeds is of universal significance. A series of research and theoretical analysis on the mechanical properties of sandwich rock were carried out by experts. Zhang et al [2] regarded rock mass with interlayer as a complete mechanical system, establishes a mechanical model of rock mass with interbeds, revealed the failure and instability mechanism of rock mass with interlayer, and studied its stability criteria. Yang and $\mathrm{Li}$ [3-5] constructed two-dimensional and three-dimensional constitutive models of interbedded salt rock mass in Cosserat medium, and carried out a series of mechanical tests of layered salt rock mass, revealed its deformation and damage mechanism, and explained many experimental phenomena. $\mathrm{Li}$ et al [6] deduced the analytical solution of stress component of composite rock mass with horizontal weak interlayer under uniaxial and triaxial compression, gave the stress distribution of interlayer, main rock mass and interface respectively, and considered the influence of confining pressure and interlayer thickness, gave the potential failure mode under uniaxial and triaxial compression.
Experts and scholars have also carried out a series of experimental to study the mechanical properties of sandwich rock. Wang et al [7] conducted triaxial tests on three sandstones and the deformation, strength and failure characteristics of three sandstones (white sandstone, red sandstone and yellow sandstone) were analysed. Attewell [8] carried out conventional triaxial loading on Penrhyn slate, and obtained cohesion and friction angle at different angles between principal stress and bedding plane, and then gave the fitting formula. Deng [9] has conducted uniaxial and triaxial compression tests of sandstone with different bedding angles. The effects of bedding angles on mechanical properties and failure modes of rock mass are analysed in detail, which provides a reference for engineering deformation and stability analysis of bedded sandstone. based on orthogonal test, Ren [10] studied the influence of temperature, chemical liquid and soaking time on mudstone interlayer, and obtained the change trend of uniaxial compressive strength and elastic modulus. Hao [11] made a series of mechanical experiments in the laboratory about self-compacted salt rock with hard rock interlayer and obtained the difference of strength and deformation of salt rock caused by interlayer. Liu [12] have carried out uniaxial and triaxial compression tests on salt rock samples with inclined mudstone interlayers. It is found that inclined interlayers have significant influence on the deformation and damage of salt rock mass. Based on the theoretical analysis of composite rock mass failure, the variation law of interfacial bond stress and its influencing factors are discussed, and the

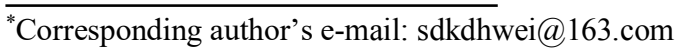


change of inclined layered salt rock mass is revealed. Characteristic and mechanism of shape and failure.

Conventional mechanical tests of yellow sandstone with mudstone interlayer have never been carried out. In this paper, natural sandstones with mudstone interlayers are selected as experimental materials and classified according to different forms of mudstone interlayers. Uniaxial compression test is carried out to measure the stress-strain curves, and the strength and crack morphology of rock are analysed, based on the failure theory of layered rock mass, which can provide some guidance for the stability of sandstone geotechnical engineering with sandwiches.

\section{Test survey}

\subsection{Test material and sample preparation}

Yellow sandstone with mudstone interlayer in Qingdao City, Shandong Province is selected as test material in this test. Mudstone distribution has different characteristics. According to mudstone interlayer dip and distribution form, it can be divided into three kinds of sandstone: sandstone with horizontal mudstone interlayer is Class I (shown in Figure 1a), mudstone interlayer distributes horizontally, but the combination of mudstone and sandstone is different, sandstone at unbonded interface and sandstone at bonded interface; sandstone with inclined mudstone interlayer is Class II (shown in Figure $1 \mathrm{~b}$ ), mudstone interlayer is inclined to a certain degree in horizontal direction, and it is bound to sandstone at the interface; sandstone with irregular distribution mudstone is. class III (shown in Figure 1c). According to standard for test methods, these sandstones were processed into samples with diameter of $50 \pm 0.3$ $\mathrm{mm}$, length of $30 \pm 0.3 \mathrm{~mm}$, and flat ends. Three kinds of sandstone with mudstone interlayer appearance features are shown in Figure 1.
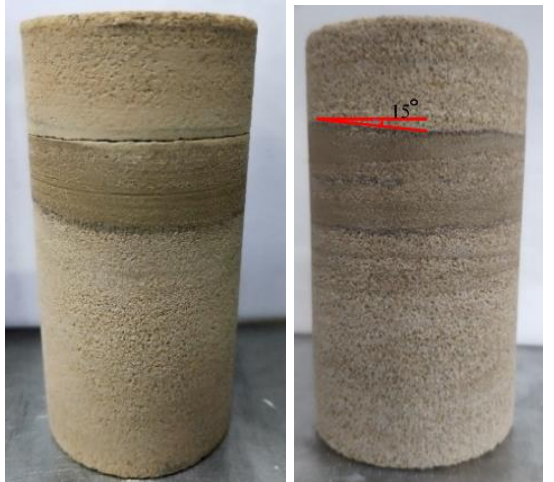

(a) Class I

(b) Class II

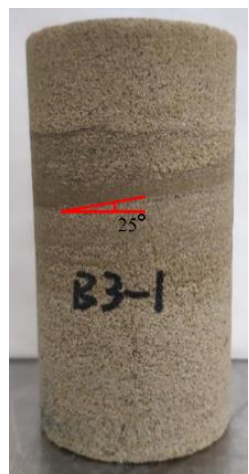

(c) Class III appearance features

\subsection{Test equipment}

The instrument used in the test was a TAW-1000 microelectro-hydraulic servo-controlled rock triaxial pressure tester (Figure 2). The parameters of the test instrument are shown in Table 1. All sandstone samples were loaded in a strained manner with a loading rate of $0.1 / \mathrm{min}$ until rock failure.

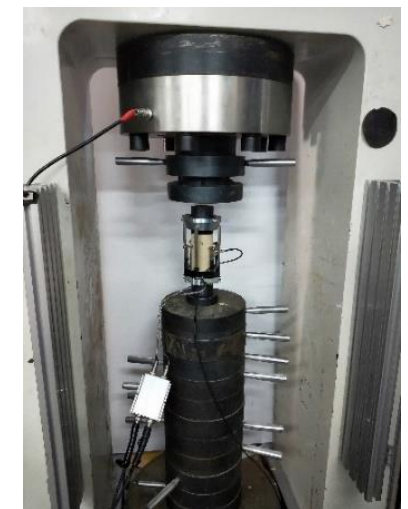

Figure 2. TAW-1000 micro-electro-hydraulic servo-controlled rock triaxial pressure tester

Table 1. Technical specifications

\begin{tabular}{cccccccc}
\hline Axial & $1000 \mathrm{KN}$ & $\begin{array}{c}\text { Confining } \\
\text { pressure }\end{array}$ & $100 \mathrm{MPa}$ & Temperature & -20 to $200^{\circ} \mathrm{C}$ & $\begin{array}{c}\text { Axial displacement } \\
\text { maximum range }\end{array}$ & $30 \mathrm{~mm}$ \\
Pressure & $0.5 \%$ & Precision & $1 \%$ & Precision & $0.2^{\circ} \mathrm{C}$ & Precision & $1 \%$ \\
\hline
\end{tabular}

\section{Compression test}

\subsection{The failure mode of different rocks}

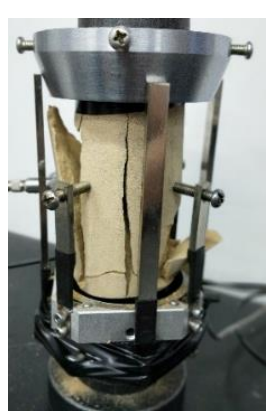

(a)sandstone

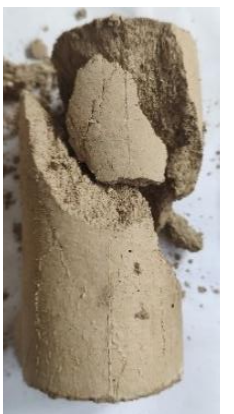

(b)mudstone

Figure 3. Failure mode of sandstone and mudstone 
The failure mode of sandstone and mudstone under uniaxial compression is shown in Figure 3. In Figure 3a, cracks parallel to the stress on the rock surface, the main crack is wider, and the crack penetrates into the interior of the rock. Cracks show obvious tension failure characteristics characteristic of tension failure. In Figure $3 \mathrm{~b}$, the damage of mudstone is obvious, main crack inclines about 45 degree to the direction of stress.

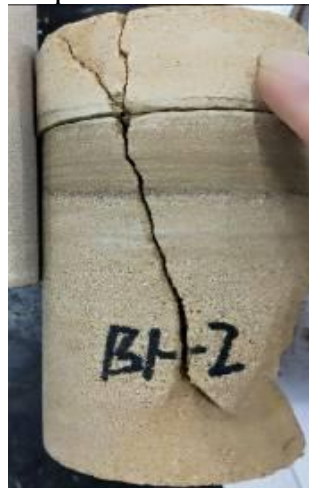

(a)Class I

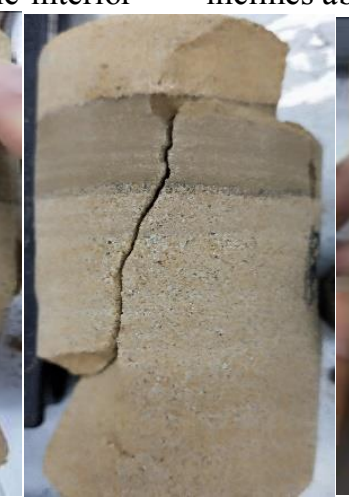

(b) Class II

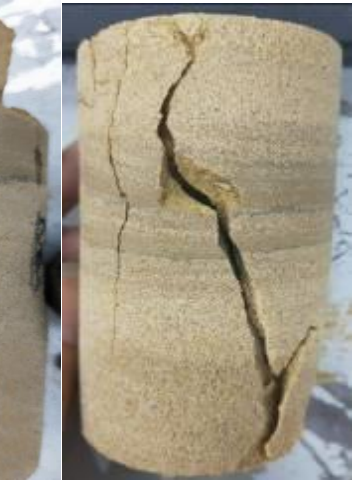

(c) Class III

Figure 4. Failure mode of sandstone with mudstone interlayer

The failure mode of sandstone with mudstone interlayer under uniaxial compression is show in Figure 4. In Figure 4a, the upper sandstone is tension failure, while mudstone interlayer and sandstone are shear failure. In Figure 4b, Class II is shear failure. In Figure 4c, crack start from the mudstone interlayer, and it stretch downward and bend upward.

\section{2 stress-strain curve}

The elastic modulus takes the stress-strain curve as the slope of the straight section (30\%-80\% peak strength), and the peak strain is the corresponding axial strain at the peak point.

$$
E=\frac{\sigma_{80}-\sigma_{30}}{\varepsilon_{80}-\varepsilon_{30}}
$$

Where, $E$ is elastic modulus, $\sigma_{80}, \sigma_{30}$ are $80 \%$ and $30 \%$ of peak stress, respectively; $\varepsilon_{80}, \varepsilon_{80}$ are $80 \%$ and $30 \%$ of peak strain, respectively.

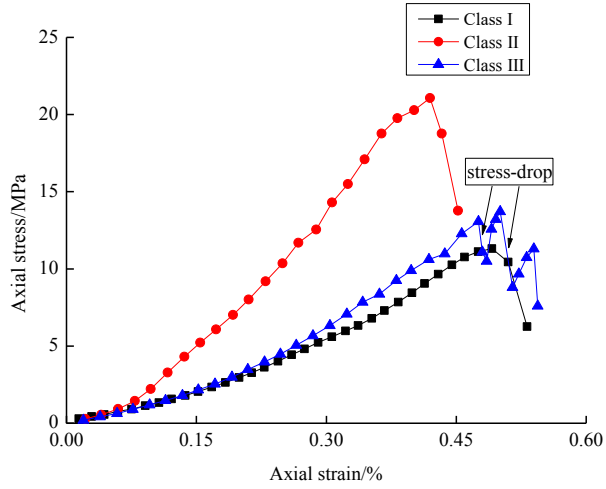

Figure 5. Each class of sanstone axial stress - strain curve

Table 2. Uniaxial compression test data for three classes of sample

\begin{tabular}{cccccccc}
\hline Category & Numbering & $\begin{array}{c}\text { Uniaxial } \\
\text { compressive } \\
\text { strength } \\
\text { /MPa }\end{array}$ & $\begin{array}{c}\text { Average } \\
\text { value/MPa }\end{array}$ & $\begin{array}{c}\text { Peak } \\
\text { strain } \\
\text { Average } \\
\text { value }\end{array}$ & $\begin{array}{c}\text { Elastic } \\
\text { modulus } \\
\text { /GPa }\end{array}$ & $\begin{array}{c}\text { Average } \\
\text { elastic } \\
\text { modulus } \\
\text { /GPa }\end{array}$ \\
\hline Sandstone & B0-1 & 32 & & 0.68 & & 10.2 & \\
Sandstone & B0-1 & 28 & 30.17 & 0.70 & 0.67 & 6.53 & 8.14 \\
Sandstone & B0-1 & 30.05 & & 0.63 & & 7.68 & \\
Class I & B1-1 & 12.11 & & $0.48 \%$ & & 2.79 & \\
Class I & B1-2 & 10.23 & 11.21 & $0.52 \%$ & 0.50 & 2.58 & 2.78 \\
Class I & B1-3 & 11.31 & & $0.49 \%$ & & 2.99 & \\
Class II & B2-1 & 21.06 & & $0.419 \%$ & & 6.06 & \\
Class II & B2-2 & 21.97 & 21.08 & $0.468 \%$ & 0.44 & 6.75 & 6.19 \\
Class II & B2-3 & 22.38 & & $0.432 \%$ & & 5.78 & \\
Class III & B3-1 & 13.70 & & $0.48 \%$ & & 3.16 & \\
Class III & B3-2 & 17.79 & 15.44 & $0.56 \%$ & 0.53 & 4.00 & 3.39 \\
Class III & B3-3 & 14.82 & & $0.55 \%$ & & 3.02 & \\
\hline
\end{tabular}

It can be seen from Table 2 , the average compressive strength of pure sandstone is $30.17 \mathrm{MPa}$. The average compressive strength of Class I is $11.21 \mathrm{MPa}$, the average compressive strength of Class II is $21.08 \mathrm{MPa}$, the average compressive strength of Class III is $15.44 \mathrm{MPa}$. As a whole, the existence of mudstone interlayer significantly reduces the strength of sandstone.
Comparing with different kind of sandstone compressive strength: pure sandstone $>$ Class II $>$ Class III $>$ Class I. The average peak strain of pure sandstone is 0.67 , the average peak strain of Class I is 0.50 , the average peak strain of Class II is 0.44 , the average peak strain of Class III is 0.53. It can be seen the existence of mudstone interlayer significantly reduces the strength of sandstone. 
The average elastic modulus of pure sandstone, class I, class II, class III are $8.14 \mathrm{GPa}, 2.78,6.19,3.39$, respectively.Comparing with different kind of sandstone elastic modulus, pure sandstone $>$ class II $>$ class III $>$ class I. Elastic modulus of class I,II,III decreased by $65.8 \%, 24.0 \%, 58.4 \%$,respectively.

\section{Stress state of interface}

\subsection{Stress state of interface of Class I}

The boundary layer between rock and rock is referred to as the interface. The sketch and coordinates of class I are shown in Figure 6 and different combinations are shown in Figure 6. At the interface between intermediate mudstone interlayer and sandstone, the upper sandstone and mudstone interlayers are freely overlapped to form a whole, and there is no other material filling between them. It means that there is no bond force between rocks. The lower sandstone and mudstone interlayer are bonded to form a whole, and there is a bond force between the rocks.

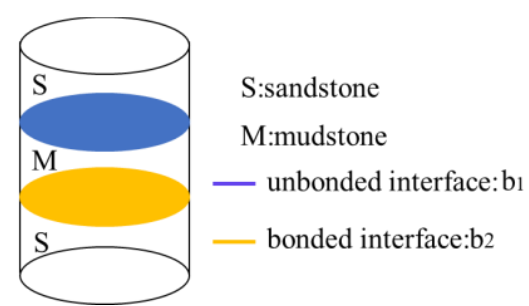

Figure 6. Layering Sketch of Class I

The rock subjected to axial stress only generates axial deformation radial deformation. For the interface $b_{1}$, because the different mechanical properties, the radial strain of mudstone interlayer and sandstone is different in interface $b_{1}$. The elastic modulus of mudstone and sandstone is related to Poisson's ratio as follows:

$$
\left\{\begin{array}{l}
\lambda_{m}>\lambda_{s} \\
E_{s}>E_{m}
\end{array}\right.
$$

Where, $\lambda_{s}$ and $\lambda_{m}$ is Poisson's ratio of mudstone and sandstone, respectively; $E_{s}$ and $E_{m}$ are the elastic modulus of sandstone and mudstone, respectively. Stress of friction restraint is inevitable at the interface, the frictional restraint of upper sandstone is tensile stress, while that of mudstone is compressive stress. The value of friction stress can be calculated:

$$
\left\{\begin{array}{l}
\sigma_{2 s}=\sigma_{3 s}=\lambda_{2} \sigma_{1}=\lambda_{3} \sigma_{1} \\
\sigma_{2 m}=\sigma_{3 m}=\lambda_{2} \sigma_{1}=\lambda_{3} \sigma_{1}
\end{array}\right.
$$

Where, $\lambda_{2}$ and $\lambda_{3}$ are the friction coefficients of rocks in $\mathrm{Y}_{0}$ and $\mathrm{Z}_{0}$ directions, respectively.

Ignoring the thickness of the interface, the sandstone and mudstone stick together. There is a bond force between the rocks in interface $b_{2}$. Sandstone is tensile stress, while that of mudstone is compressive stress. Two kinds of stresses are equal in value and opposite in direction. According to the strain coordination relationship, considering the continuous deformation condition and the static equilibrium condition, the additional stress in the horizontal direction at the bonding interface was deduced by Xian [1].

$$
\sigma_{2 s}=\sigma_{2 n}=\sigma_{3 s}=\sigma_{3 n}=\frac{E_{s} \lambda_{m}-E_{m} \lambda_{s}}{E_{s}\left(1-\lambda_{m}\right)+E_{m}\left(1-\lambda_{s}\right)} \sigma_{1}
$$

According to lateral strain constraints on interface $b_{2}$, mudstone is subjected to triaxial compression stress and sandstone is subjected to triaxial compression-tension stress. Although same lithology of Upper and lower sandstone, sandstone at unbonded interface is failure before sandstone at bonded interface. The compressive strength of lower sandstone is more than upper sandstone. According to Mohr-Coulomb Criterion of Linear Type, we can obtain:

$$
\lambda_{3}>\frac{E_{s} \mu_{m}-E_{m} \mu_{s}}{E_{s}\left(1-\mu_{m}\right)+E_{m}\left(1-\mu_{s}\right)}
$$

When $E_{s}=8.0 \mathrm{GPa}, E_{m}=4 \mathrm{GPa}, \mu_{m}=0.38, \mu_{s}=0.25$, we can calculate the friction coefficient $\lambda_{3}>0.256$.

Sandstone has higher strength and mudstone interlayer has lower strength. Influenced by interlayer constraints, transverse compressive stress increases the strength of mudstone and horizontal tensile stress reduces sandstone Strength, Sandstone failure precedes mudstone occurs. Although combination mode is different between $b_{1}$ and $b_{2}$, all sandstone in the interface is subjected to tension stress in direction. According to Eq. (5), $\lambda_{3}>0.256$.

\subsection{Stress state of interface of Class II}

The complex stress-strain relationship makes it possible for cracks to nucleate and propagate firstly at the interface [13]. Stress at rock interface is a key problem in strength analysis of rock with interlayer. For the stress analysis at the interface of inclined rock mass, Xian [1] takes into account the interlayer bonding stress condition under the constraint of interlayer deformation., coordinate axes are transformed firstly. Then, according to the principle of mechanical superposition, the stress solutions of different lithologic rocks at the interface of inclined rock mass are obtained. For inclined layered sandstone in geodetic coordinate $\mathrm{x}_{0} \mathrm{y}_{0} \mathrm{z}_{0}$, the expression of interfacial bond stress in local coordinate $Z_{x y}$ is obtained by coordinate transformation.

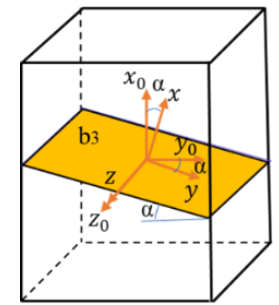

Figure 7. Layering sketch of inclined interface $b_{3}$

The stress matrix of rock in geodetic coordinate is as follows: 


$$
\sigma=\left(\begin{array}{ccc}
\sigma_{x_{0}} & & \\
& \sigma_{y_{0}} & \\
& & \sigma_{z_{0}}
\end{array}\right)
$$

We can obtain stress components at declined interface by coordinate transformation,

$$
\left\{\begin{array}{l}
\sigma_{x s}=\sigma_{\mathrm{x}} \\
\sigma_{y s}=-k_{1} \sigma_{z}+\left(\sigma_{y}+k_{2} \sigma_{y}\right)-k_{3} \sigma_{x} \\
\sigma_{z s}=\left(\sigma_{z}+k_{2} \sigma_{z}\right)-k_{1} \sigma_{y}-k_{3} \sigma_{x} \\
\tau_{x y s}=\tau_{\mathrm{xy}} \\
\tau_{y z s}=k_{4} \tau_{\mathrm{yz}} \\
\tau_{z x s}=\tau_{z x}
\end{array}\right.
$$

Where: $k_{1}=\frac{2 E_{m} E_{s}\left(\lambda_{m}-\lambda_{s}\right)}{\left(E_{s}+E_{m}\right)^{2}-\left(E_{m} \lambda_{s}+E_{s} \lambda_{C}\right)^{2}}$

$$
k_{2}=\frac{E_{s}{ }^{2}\left(1-\lambda_{m}{ }^{2}\right)-E_{m}{ }^{2}\left(1-\lambda_{s}{ }^{2}\right)}{\left(E_{s}+E_{m}\right)^{2}-\left(E_{m} \lambda_{s}+E_{s} \lambda_{m}\right)^{2}}
$$

$$
k_{3}=\frac{E_{s}^{2}\left(\lambda_{m}{ }^{2}+\lambda_{m}\right)-E_{m}{ }^{2}\left(\lambda_{s}{ }^{2}+\lambda_{s}\right)+E_{m} E_{s}\left(\lambda_{m}-\lambda_{s}\right)}{\left(E_{m}+E_{s}\right)^{2}-\left(E_{m} \lambda_{s}+E_{s} \lambda_{m}\right)^{2}}
$$$$
k_{4}=\frac{2 E_{s}\left(1+\lambda_{m}\right)}{E_{m}\left(1+\lambda_{s}\right)+E_{s}\left(1+\lambda_{m}\right)}
$$

$$
k_{5}=\frac{2 E_{m}\left(1+\mu_{s}\right)}{E_{m}\left(1+\mu_{s}\right)+E_{s}\left(1+\mu_{m}\right)}
$$

Where, $m$ is mudstone, $s$ is sandstone. $k_{1}, k_{2}, k_{3}$, $k_{4}, k_{5}$ is all material constant.

Sandstone stress components at inclined interface $b_{3}$ is:

$$
\sigma_{s}=\left(\begin{array}{lll}
\sigma_{x s} & \tau_{x y s} & \tau_{x z s} \\
\tau_{y x s} & \sigma_{y s} & \tau_{y z s} \\
\tau_{z x s} & \tau_{z y s} & \sigma_{z s}
\end{array}\right)
$$

Taking dip angle of $\alpha=15^{\circ}$,we can obtain material constant by calculated $\mathrm{k}_{1}=0.195, \mathrm{k}_{2}=0.311, \mathrm{k}_{3}=0.191$, $\mathrm{k}_{4}=1.377, \mathrm{k}_{5}=0.623$.

Sandstone stress components values at interface $b_{3}$ is:

$$
\sigma_{s}=\left(\begin{array}{ccc}
9.33 & -2.50 & 0 \\
-2.50 & -0.90 & 0 \\
0 & 0 & -1.91
\end{array}\right)
$$

From the stress matrix, mudstone is subjected to triaxial compression-tension stress and shear stress in $x y$ direction. Tension stress $0.90 \mathrm{MPa}<1.91 \mathrm{MPa}$.

\subsection{Stress state of interface of Class III}

Sandstone with irregular distribution mudstone is Class III. According to the experimental phenomena, cracks occur in the boundary of mudstone and sandstone with a large inclination angle. Taking dip angle of $\alpha=25^{\circ}$, sandstone stress components values at interface with a large inclination angle is:

$$
\sigma_{s}=\left(\begin{array}{ccc}
8.20 & -3.83 & 0 \\
-3.83 & -0.78 & 0 \\
0 & 0 & -1.92
\end{array}\right)
$$

It is obvious that the shear stress of sandstone with large inclination angle is much greater than that of sandstone with small inclination angle, which is consistent with the experimental phenomena. The stress state of sandstone interbeds is determined by the stratified dip angle in the local area of mudstone interlayer. With the increase of the dip angle, the shear stress of interlayer increases. Horizontal additional tensile stress and shear stress promote the fracture of mudstone interlayer with larger dip angle, but all parts of rock remain intact after cracking. At save time, there is a stress drop in the curve, crack causes a softening process similar to that of sandwich material. When the load continues to increase, the sandstone and mudstone grains in the composite rock stratum are distorted and squeezed, and the stress redistribution in the composite rock stratum is realized.

\section{Conclusion}

Uniaxial compression test about different sandstone is carried out, the main findings can be summarized as follows:

(1) Mudstone interlayer has a significant impact on the failure of sandstone, which makes interlayer and sandstone show different fracture patterns.

(2) The initiation of interbedded mudstone rocks causes a softening process similar to that of interbedded materials, resulting in stress drop.

(3) Different kind of sandstone compressive strength: class II $>$ class III $>$ class I. different kind of sandstone elastic modulus, pure sandstone $>$ class II $>$ class III $>$ class I

\section{References}

1. Xian, X.F., Tan X.S. (1989) Failure Mechanism of Stratified Rock Mass. Chongqing University Press, Chongqing.

2. Zhang, D.L., Wang Y.H., Qu T.H. (2000) Influence analysis of interband on stability of stratified rock mass. Chinese Journal of Rock Mechanics and Engineering, 19(2): 140-144.

3. Yang, C.H., LI Y.P. (2005) The expanded Cosserat medium constitutive model for laminated salt rock. Chinese Journal of Rock Mechanics and Engineering, 24(23): 4226-4232.

4. Li, Y.P., Yang, C.H. (2006) Three-dimensional expanded Cosserat medium constitutive model for laminated salt rock. Rock and Soil Mechanics, 27(4): 509-513. 
5. Li, Y.P., Liu, J., Yang, C.H. (2006) Influence of mudstone interlayer on deformation and failure characteristics of salt rock. Chinese Journal of Rock Mechanics and Engineering, 2006, 25(12): $2463-$ 2466.

6. Li, J.G. (2015) Journal of Qingdao University of Science and Technology[D]. Study on strength and creep characteristics of composite rock mass with incliend soft-weak interlayer.

7. Wang, Y.F., Su, H.,Wang, L.P. et al(2019)Study on the difference of deformation and strength characteristics of three kinds of sandstone. Journal of China Coal Society.

8. Jaeger J C. (1960) Shear failure of anisotropic rock. Geol Mag. ,97: 65-72.

9. Deng, H.F., Wang, W.; Li, J.L. et al. (2018) Experimental study on anisotropic characteristics of bedded sandstone[J]. Chinese Journal of Rock Mechanics and Engineering,37(01):112-120.

10. He, T.S., Geng, Y.D., Chen, Y.D. (2017) Experimental investigation on mechanical bahavious of salt rock containing brittle-hard interlayers. Rock and Soil Mechanics. 38(11):3119-3126.

11. Ren, S., Wen, Y.J. et al (2010) Research on Softening in Mudstone Interlayer. Rock and Soil Mechanics.34(11):

12. Liu, Wei, Li, Y.P., Yin, D.L.et al. (2013) Analysis of deformation and fracture characteristics of salt rock with tilted interlayer. Rock and Soil Mechanics. 34(03):645-652

13. Xu, J.Q. (2006)Interface Mechanics. The Science Publishing Company, Beijing. 